\title{
Hierarchical FAU-type Hafnosilicate Zeolite as a Robust Lewis Acid Catalyst for Catalytic Transfer Hydrogenation
}

Bo Tang ${ }^{1}$, Shuang Li ${ }^{1}$, Wei-Chao Song ${ }^{1}$, En-Cui Yang ${ }^{1, *}$, Xiao-Jun Zhao ${ }^{1, *}$, Naijia Guan ${ }^{2}$, and Landong $\mathrm{Li}^{2}$,*

1 Key Laboratory of Inorganic-Organic Hybrid Functional Material Chemistry, Ministry of Education, Tianjin Key Laboratory of Structure and Performance for Functional Molecules, Tianjin Normal University, 393\# Binshui West Road, Xiqing District, Tianjin 300387, China

2 School of Materials Science and Engineering \& National Institute for Advanced Materials, Nankai University, 38\# Tongyan Road, Jinnan District, Tianjin 300350, China

*Corresponding email: encui_yang@163.com (E.-C. Yang) xiaojun_zhao15@163.com (X.-J. Zhao)

lild@nankai.edu.cn (L. Li)

Page: S1-S9

Figure: S1-S11

Table: S1 
The substrate conversion, product selectivity and yield were calculated as follows:

Substrate conversion $($ mol $\%)=\frac{\text { initial moles }- \text { final moles }}{\text { initial moles }} \times 100 \%$

Product yield $($ mol $\%)=\frac{\text { moles of product formed }}{\text { initial moles of substrate }} \times 100 \%$

Product selectivity $($ mol $\%)=\frac{\text { product yield }}{\text { substrate conversion }} \times 100 \%$

Carbon balance $($ mol $\%)=\frac{\text { moles of products formed }}{\text { moles of substrate converted }} \times 100 \%$

Reaction rate constants: In the kinetic study, the points of $\ln \left(\mathrm{C}_{\text {ethyl levulinate }}\right)$ as a function of time at the initial reaction (conversions not exceeding 20\% within $1 \mathrm{~h}$ ) were recorded, followed by making the straight lines with the best fitting of the points. The rate constants $(\mathrm{k})$ are calculated as the slopes of the straight lines determined at the four different temperatures.

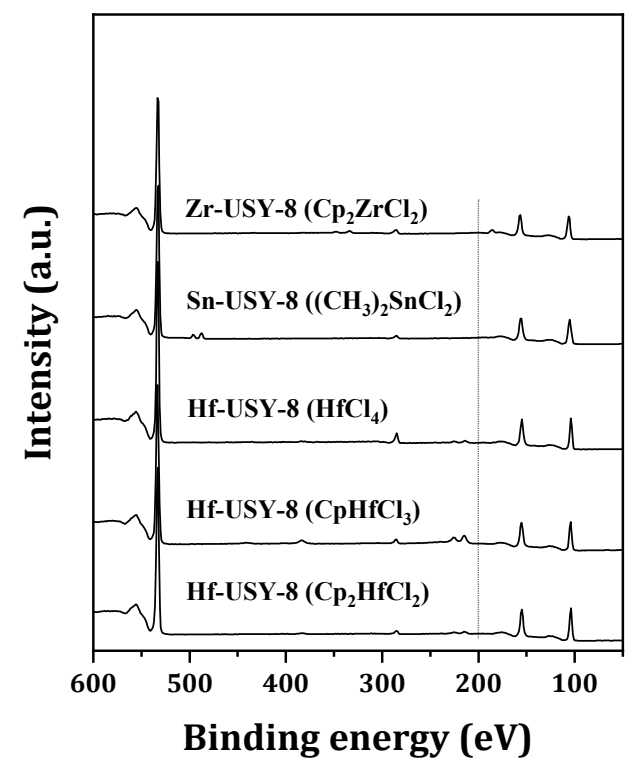

Figure S1 XPS of Sn-, Zr- and Hf-USY-8 prepared from different precursors

As shown, there are no signals occurring at binding energies of ca. $200 \mathrm{eV}$ for all the samples, ruling out the existence of residual chloride species. Such results suggest that the metal precursors can decompose completely for heteroatoms incorporation under the identical thermal conditions employed in this work. 


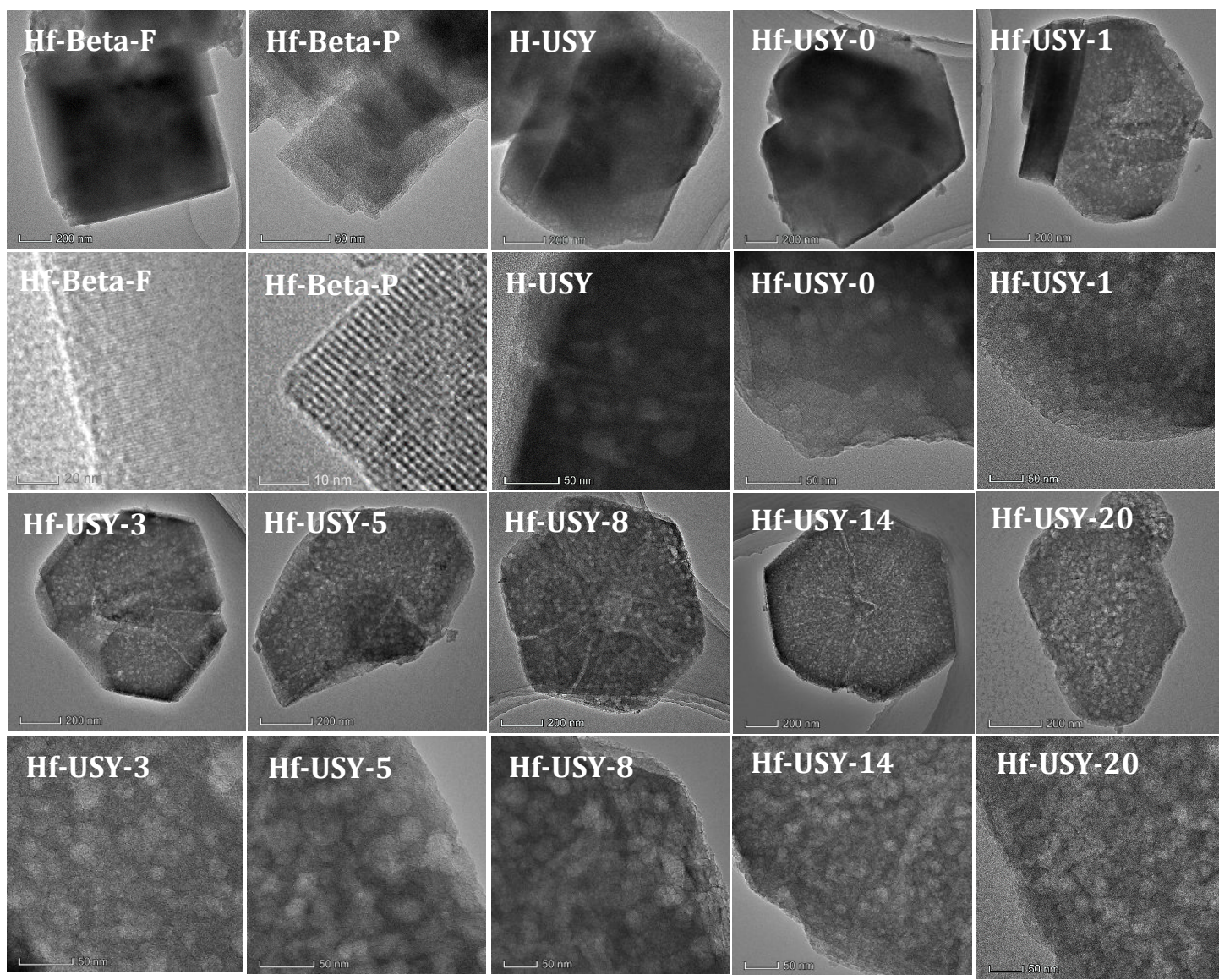

Figure S2 TEM and HR-TEM images of Hf-containing zeolites

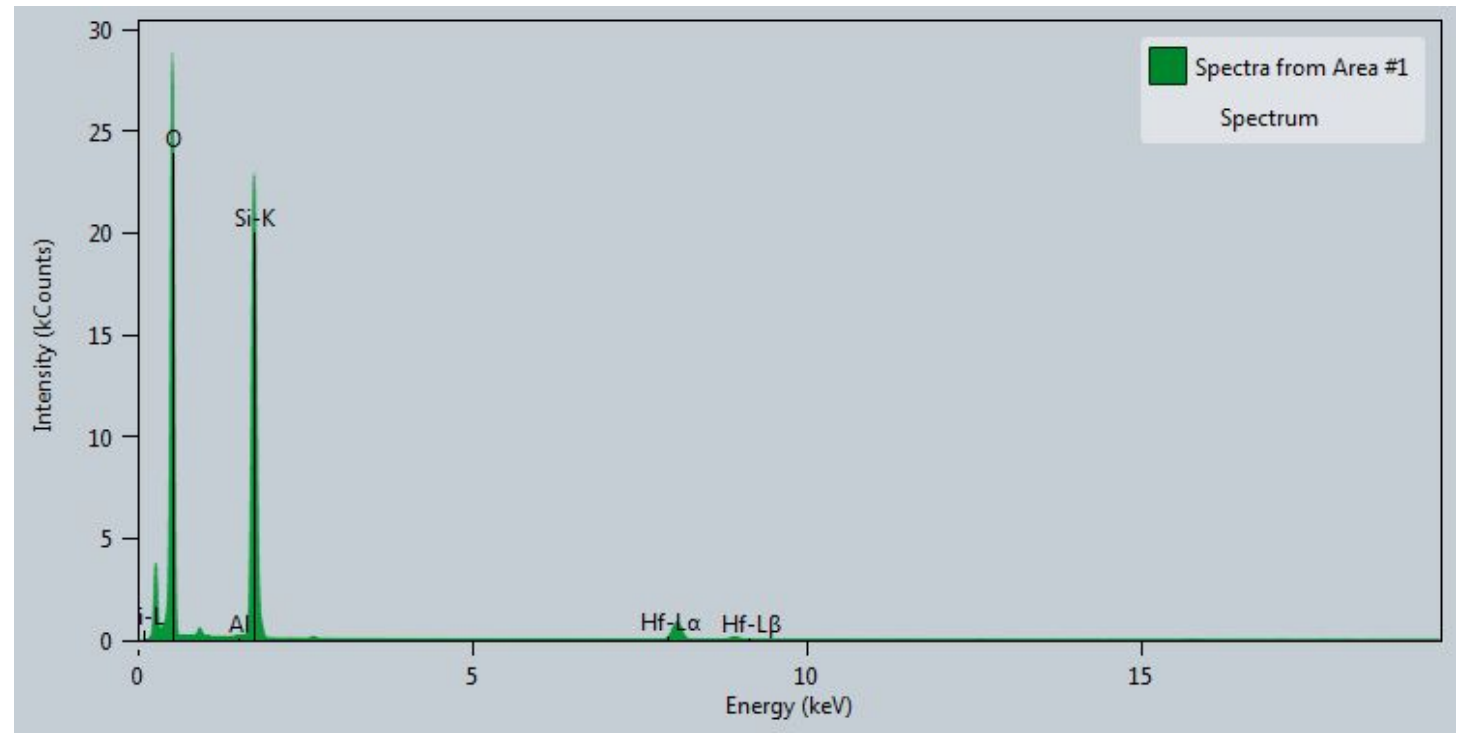

Figure S3 EDX analysis of Hf-USY-8 zeolite 
(a)

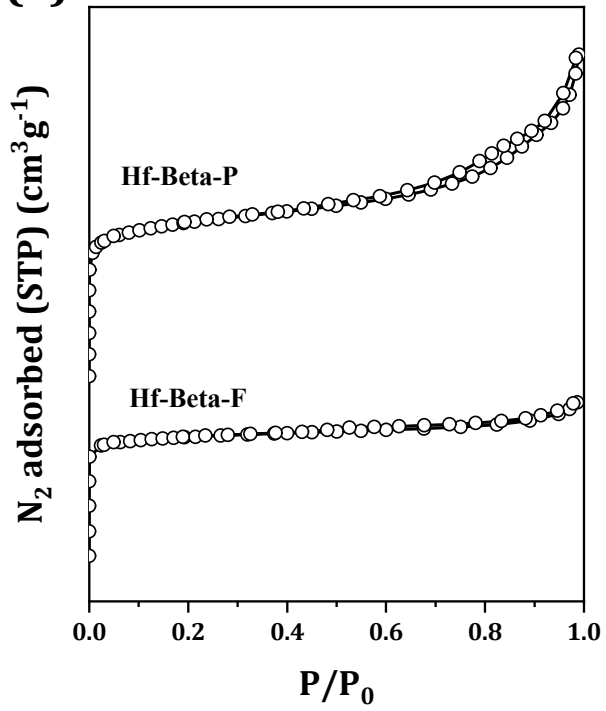

(b)

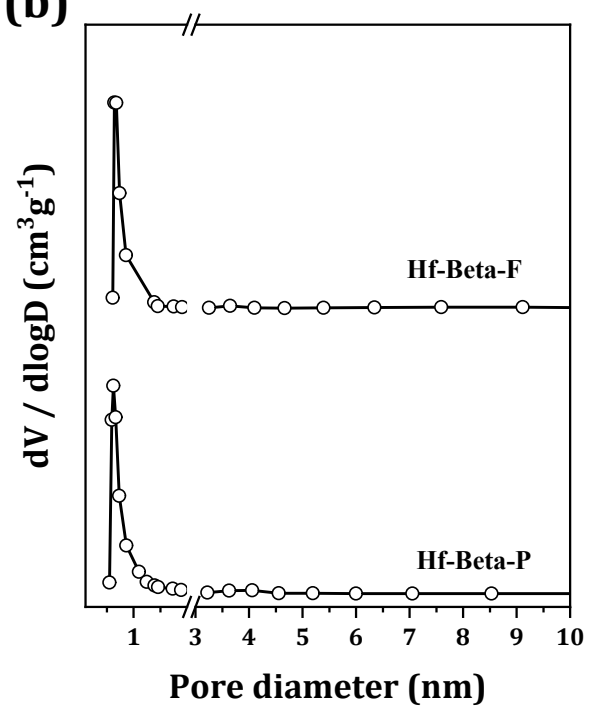

Figure S4 $\mathrm{N}_{2}$ adsorption-desorption isotherms (a) and pore size distribution curves (b) of Hf-Beta-P and Hf-Beta-F.

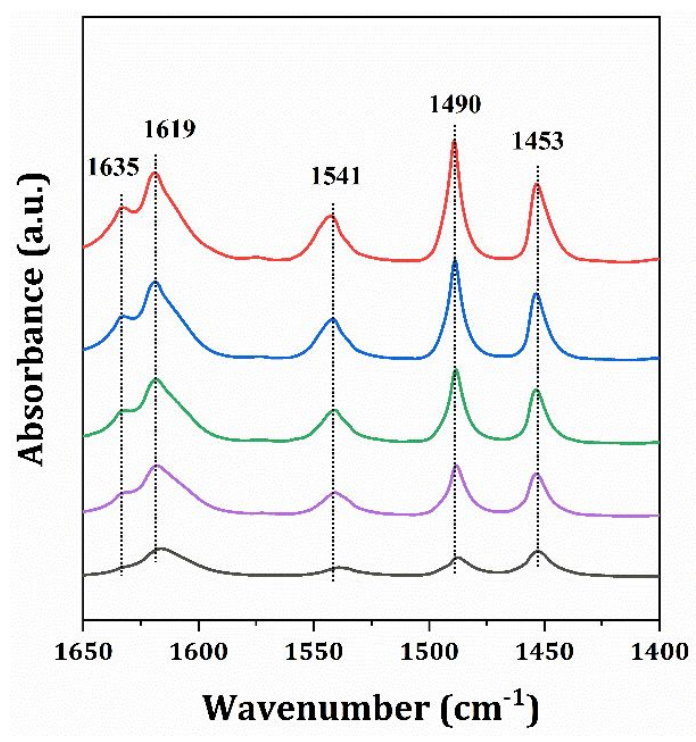

Figure S5 FT-IR spectra of pyridine adsorption on H-USY after desorption at 423, $473,523,573$ and $673 \mathrm{~K}$ (from top to bottom), respectively. 


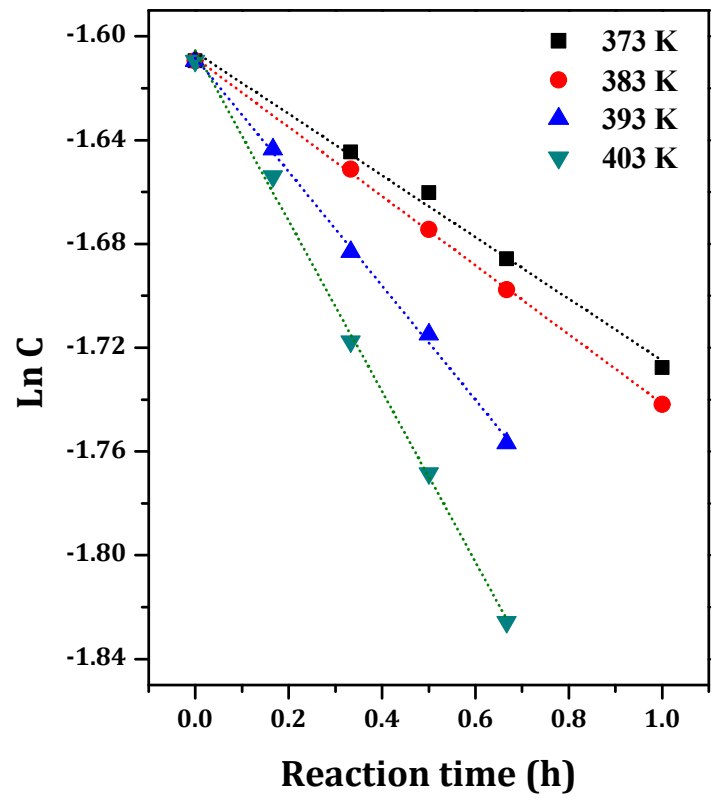

Figure S6 Kinetic plots of ethyl levulinate transfer hydrogenation over Hf-USY-8 zeolite.

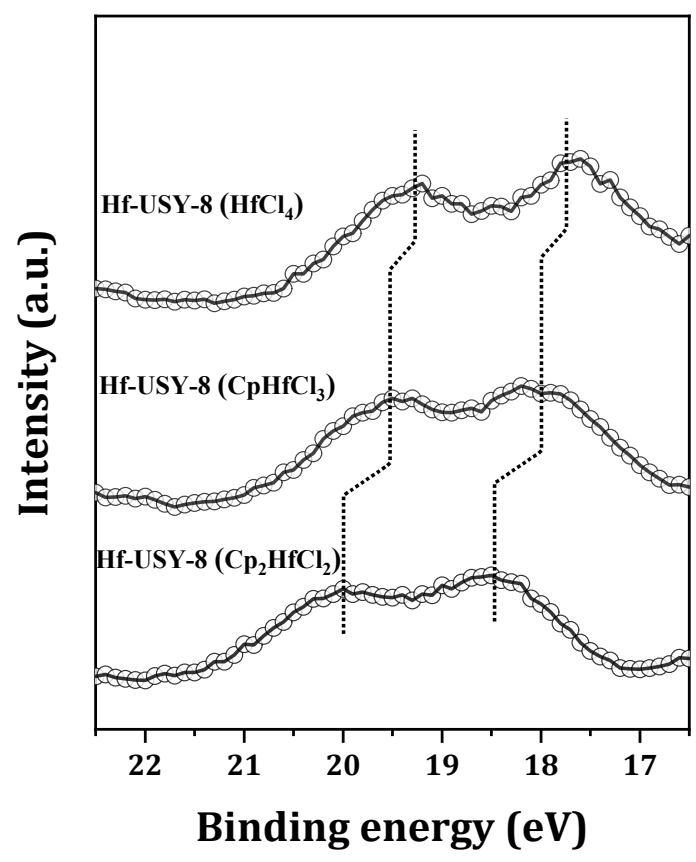

Figure S7 Hf 4f XPS of Hf-USY-8 synthesized from different Hf precursors 


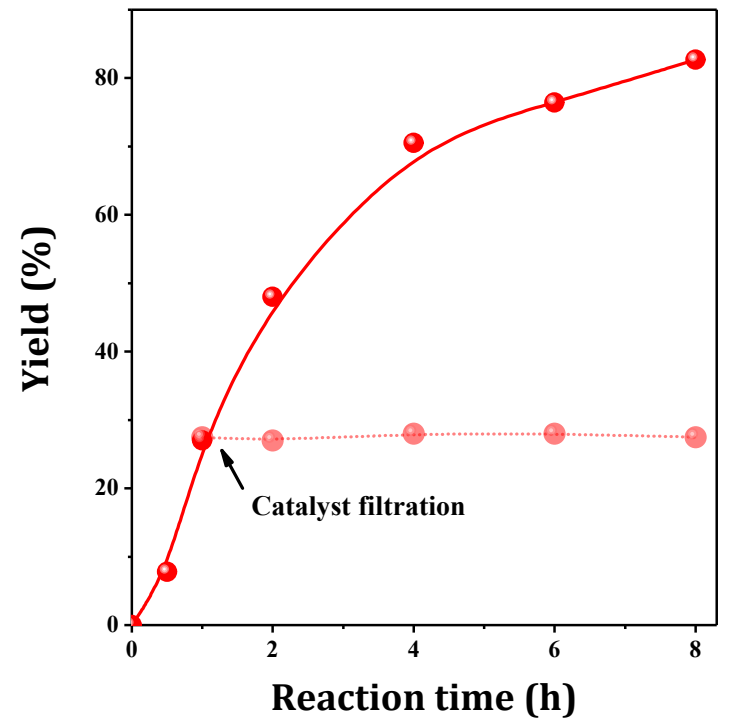

Figure S8 Time-dependent $\gamma$-valerolactone yield in the transfer hydrogenation of ethyl levulinate over Hf-USY-8. Reaction conditions: ethyl levulinate, $1 \mathrm{mmol}$; alcohol, $5 \mathrm{~mL}$; catalyst, $100 \mathrm{mg}$; $\mathrm{N}_{2}$ pressure, $1.0 \mathrm{Mpa}$; Temperature, $393 \mathrm{~K}$; Time, 8 $\mathrm{h}$ 
(a)

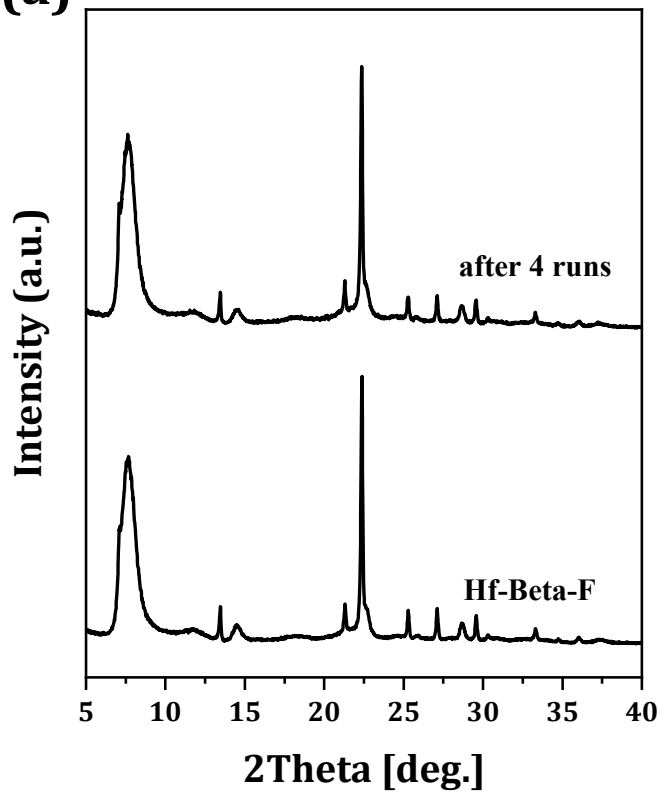

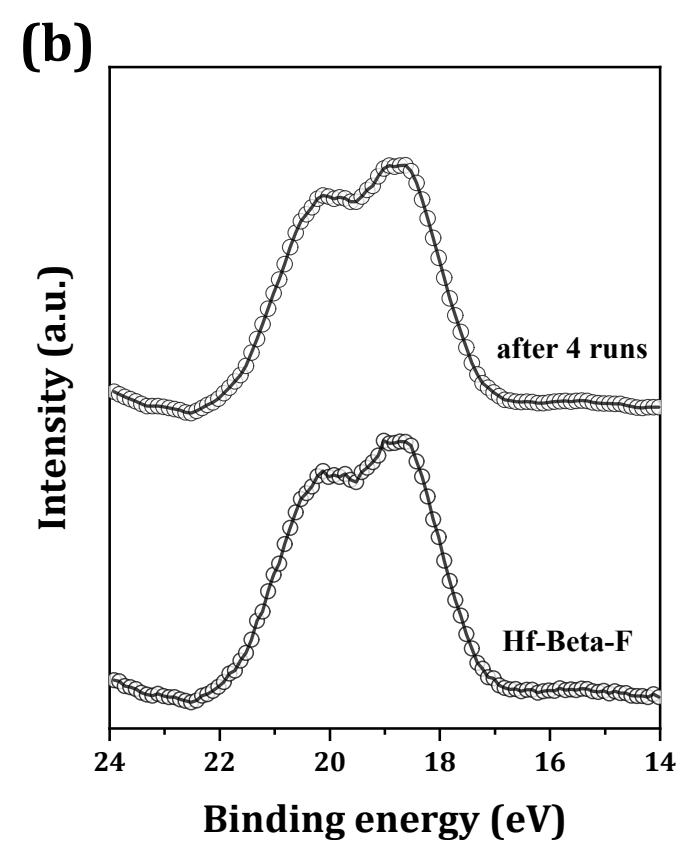

(b)

Figure S9 XRD (a) and XPS (b) of Hf-Beta-F before and after catalytic recycles

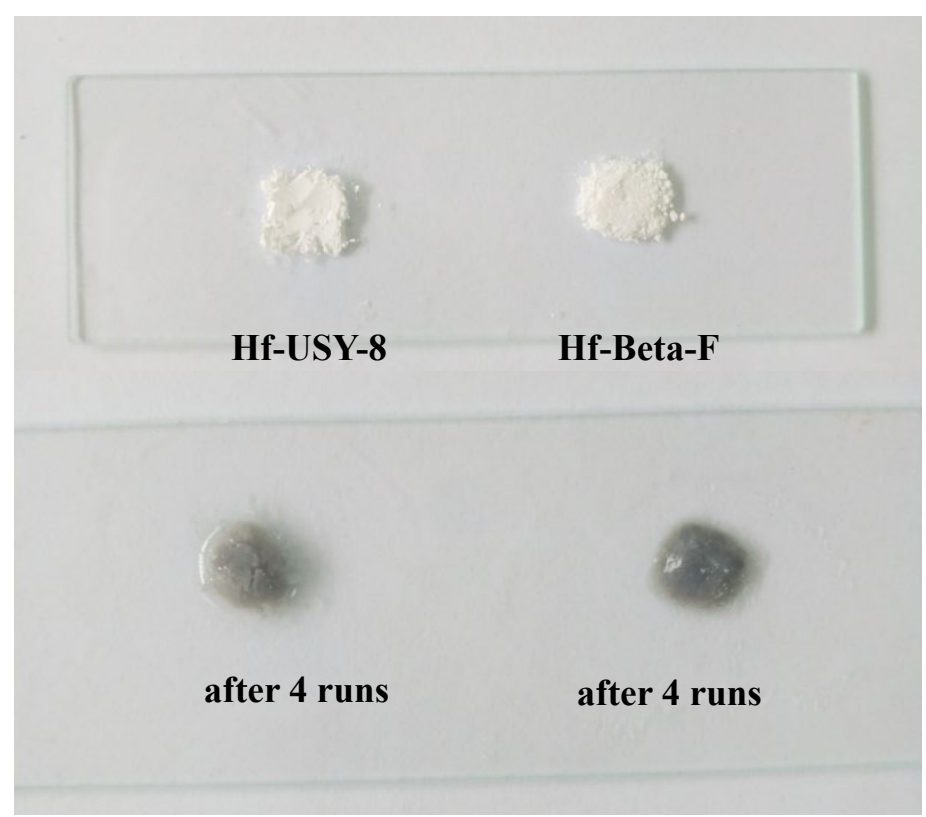

Figure S10 Pictures of Hf-USY-8 and Hf-Beta zeolites before and after catalytic recycles 

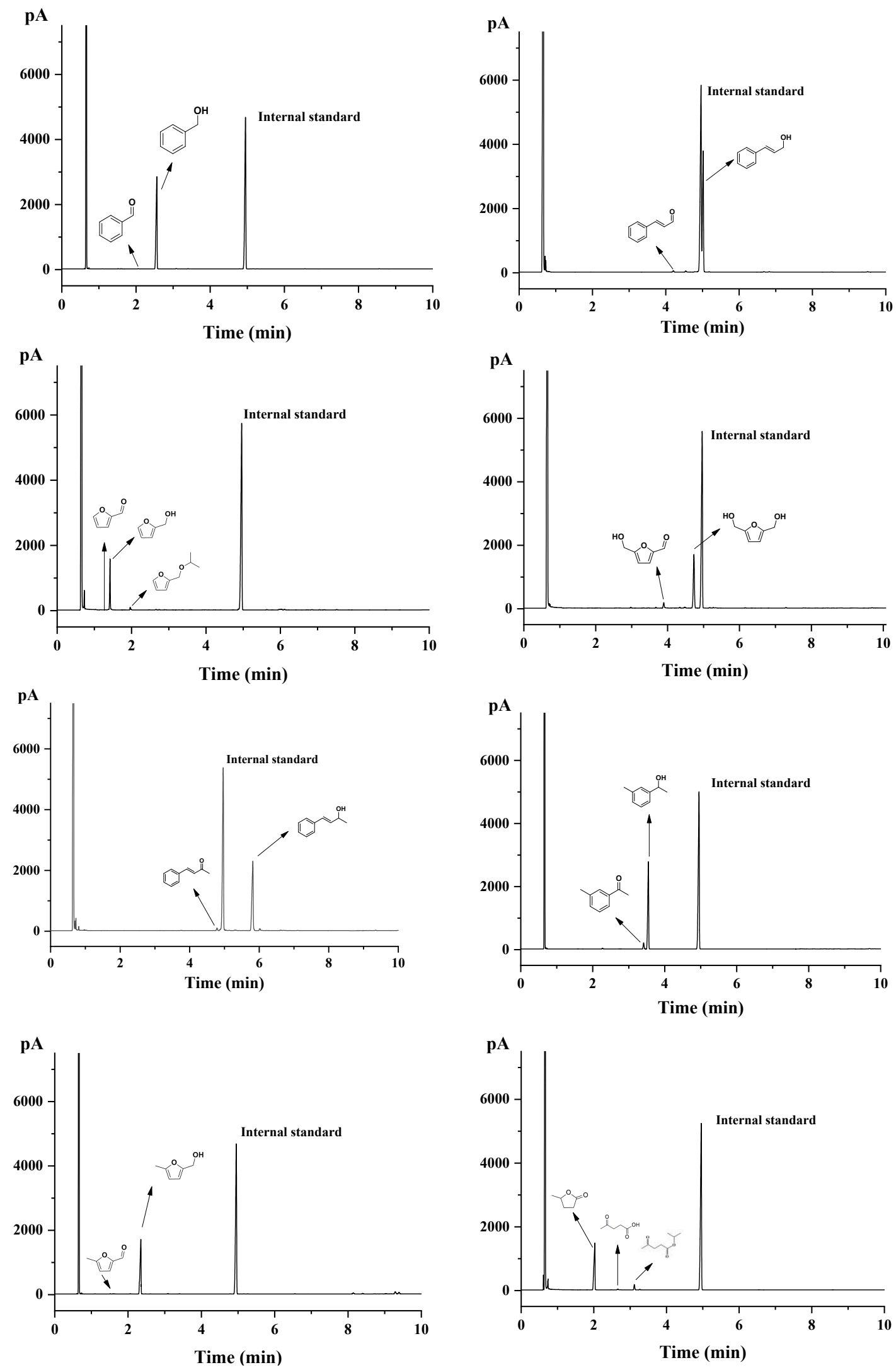

Figure S11 Typical chromatograms of reaction mixtures in Table 3 
Table S1 Physicochemical properties of H-USY-8 before and after catalytic recycles

\begin{tabular}{ccc}
\hline Sample & Surface area & Pore volume \\
\hline Hf-USY-8 as synthesized & 953 & 0.559 \\
Hf-USY-8 after 4 runs & 815 & 0.532 \\
Hf-USY-8 regenerated & 961 & 0.560 \\
Hf-Beta-F & 498 & 0.313 \\
Hf-Beta-F after 4 runs & 371 & 0.263 \\
\hline
\end{tabular}

